\title{
The whole truth about botulinum toxin - a review
}

\author{
Henryk Witmanowski ${ }^{1}$, Katarzyna Błochowiak ${ }^{2}$
}

${ }^{1}$ Department of Plastic, Reconstructive and Aesthetic Surgery, Collegium Medicum in Bydgoszcz, Nicolaus Copernicus University in Torun, Poland

${ }^{2}$ Department of Oral Surgery and Periodontology, Poznan University of Medical Sciences, Poznan, Poland

Adv Dermatol Allergol 2020; XXXVII (6): 853-861

DOI: https://doi.org/10.5114/ada.2019.82795

\begin{abstract}
Treatment with botulinum toxin is widely viewed as safe, effective and largely devoid of serious side effects. There are two classes of Botox-related adverse events - transient and benign events, and potentially serious events. The aim of this study was to provide an overview of Botox-related side effects and advise potential management and preventive strategies. Benign side effects are well-localized, reversible and self-limited complications which develop within a few days of the injection, and they usually disappear without any treatment. The aesthetic and functional adverse effects are associated with different muscle responses to botulinum toxin or with misplacement of botulinum toxin. The serious events are sequelae due to the systemic spread of toxin leading to botulism.
\end{abstract}

Key words: botulinum toxin, plastic surgery, aesthetic surgery.

\section{Introduction}

Botulinum toxins are among the most toxic poisons, with a wide spectrum of both therapeutic and cosmetic indications for use. Injections with botulinum toxin are effective for many clinical disorders that involve involuntary muscle activity or increased muscle tone. It is also the most common cosmetic procedure performed worldwide, with estimates of nearly 3 million injections per year [1]. Dynamic wrinkles are the main cosmetic indications for the use of botulinum toxin. The action of botulinum toxins is a result of their potent ability to inhibit neurotransmitter release. There are multiple serotypes of botulinum toxins A-G varying with the molecular size, biosynthesis and cell mechanisms. Type A (BoNT-A) is the most potent and it is used the most commonly. At present, there are three BoNT-A products available worldwide: abobotulinumtoxinA (Dysport), onabotulinumtoxinA (Botox), and incobotulinumtoxinA (Xeomin) [2]. Treatment with botulinum toxin is widely viewed as safe, effective and largely devoid of serious side effects. Sequelae that can occur at any site after a botulinum toxin injection include pain, edema, erythema, ecchymosis, and shortterm hypesthesia. These do not differ from side-effects after other types of injections. When botulinum toxin is used properly, the incidence of these complications is low. There are no long-term or life-threatening adverse effects related to botulinum toxin treatment for any cos- metic indications. Moreover, the risk of possible complications can be reduced by means of a thorough analysis of the patient's medical history and the use of the appropriate dose and technique for the injection. On the other hand, injections with botulinum toxin may be responsible for more severe side-effects. They are associated with systemic and generalized botulinum toxin diffusion. There are two classes of adverse events reported so far - transient and benign events, and potentially serious events [1-6]. It seems to be important to gain appropriate knowledge about all possible adverse effects, their mechanisms and ways of preventing them.

The aim of this study was to provide an overview of the possible complications and side effects that can arise after cosmetic use of botulinum toxin and advise potential management and preventive strategies.

\section{Transient and benign side-effects}

Transient and benign side effects are usually well-localized, reversible and self-limited complications which develop within a few days of the injection, and they usually disappear without any additional treatment. Most of them are injection site adverse effects. Ecchymosis or bruising often results from injuring a blood vessel at the site of the injection and most commonly occurs around the ocular area. Mild bruising affects $11 \%$ to $25 \%$ of pa-

Address for correspondence: Katarzyna Błochowiak PhD, DDS, Department of Oral Surgery and Periodontology, Poznan University of Medical Sciences, 70 Bukowska St, 60-812 Poznan, Poland, e-mail: kasia@naszdentysta.com.pl Received: 1.12.2018, accepted: 6.01.2019. 
tients [6]. A more severe complication related to blood vessel injuries is hematoma. It can develop immediately after injection and can last for a longer period. The hematoma usually requires prevention against abscess formation with antibiotics. Managing this with simple tamponade at the time of the bleeding can decrease the size of the bruise formed or even prevent it from appearing. Pressure and ice packs on the injection site should be applied immediately after blood vessel injuries to reduce ecchymosis and bruising. Preventative methods for bruising include applying ice to the site prior to the injection, causing vasoconstrictive effects, and also ensuring that the patient is not taking any blood-thinning medications such as warfarin or aspirin, and does not have any underlying clotting problems. Precautions and suggested recommendations for patients to avoid medications that inhibit clotting such as vitamin E, aspirin, and nonsteroidal anti-inflammatory drugs (NSAIDs) for a period of 10-14 days prior to treatment minimize the incidence of bruising [5]. Moreover, herbal remedies, such as ginseng, ginkgo, and high doses of garlic, may be associated with higher rates of bruising [6].

Another injection site complication is localized pain, which is associated with the needle puncturing the skin. Pain that is greater than normal is often associated with using the incorrect needle size. Topical anesthetic creams can be used to minimize pain associated with the injection. Suggested and safe techniques include having the needle bevel up and injecting slowly [7]. Pain can also be minimized by using a preservative containing saline solution to dilute the botulinum toxin [4]. The reduction of injection pain may be the result of the higher $\mathrm{pH}$ of the preservative-enhanced saline solution [6]. Another localized skin reaction, namely dry skin and subsequent flakiness, can occur in some patients [6]. It has been hypothesized that localized skin dryness may be due to decreased sweat gland activity. On rare occasions, a localized injection site side effect may manifest as an injection trauma.

Infection is a rare possible injected site complication and can occur with any procedure that breaches the skin barrier. Infection can develop at any injection site if the area is not adequately prepared. To prevent infection, the injection site should be cleaned with an antiseptic solution with all makeup residue removed. To avoid contamination, an aseptic technique should be employed [6]. Additionally, after the treatment, the patient should be instructed not to touch the area until it has healed or apply makeup for at least $6 \mathrm{~h}$ to allow the open injection sites to close [4]. To manage infections, a course of antibiotics and close monitoring of the area is essential to ensure that an abscess does not form, which could potentially result in a scar. A case of psoriasiform eruption related to botulinum toxin injections has been described which resolved spontaneously within 5 months following treatment [6]. This manifestation can be an effect of the disturbances between botulinum toxin and nerve endings in nerve-rich skin areas. Psoriatic skin contains more nerve fibers, and has increased levels of sensory nerve-derived calcitonin gene-related peptide (CGRP) and substance $P$ (SP). Botulinum toxin is a known inhibitor of CGRP/SP release, and can serve as a supplemental therapy of psoriasis. The manifestation of psoriasiform eruption after use of botulinum toxin is a result of the improper reaction between nerve fibers and botulinum toxin [7].

Headache is a common short-term side effect, as the toxin initially causes muscle spasm and then complete paralysis. Another explanation for mild headaches is the needle hitting the periosteum or deep muscle hematomas [6]. Moreover, the stress of the injections themselves may be an important factor in patients who experience transient headaches. Headaches can occur with facial injections. Most of these headaches are mild and resolve a few days after treatment. There are also reports of idiosyncratic severe headaches lasting two to 4 weeks [8]. Nonsteroidal anti-inflammatory drugs or opioids may be used to manage headaches based on severity [8]. Paresthesia or dysesthesia in the treatment area is rare, and may be caused by nerve trauma [8]. Hypoesthesia may also be related to the localized antinociceptive properties of botulinum toxin, which has been suggested by preliminary in vivo and in vitro data [6]. Its range and severity depend on the type of nerve branch. Headaches and hypoesthesia usually last for 24-48 h after botulinum toxin treatment. Dry mouth sensation and flu-like mild malaise can also occur after botulinum toxin injections [6]. Bruising and headaches can be a common complication after botulinum toxin used in the treatment of rosacea flushing $[9,10]$.

Botulinum toxin can result in allergic reactions. These reactions consist of a wide spectrum of symptoms, ranging from edema, erythema or redness limited to the site of injection, through diffuse erythema and generalized urticaria to anaphylactic shock [8]. Erythema should settle within $24 \mathrm{~h}$; if it lasts any longer, an antihistamine can be helpful. If it is an anaphylactic reaction, then this is a medical emergency. In such circumstances, treatment with an adrenalin injection and continual monitoring of the patient should be implemented. To help to prevent allergic reactions, a thorough medical history should be taken to rule out previous allergic reactions to other brands of botulinum toxin, and practitioners should exercise extra caution in treating someone with multiple allergies, as they may have a higher risk of being allergic to botulinum toxin.

In addition to injection site complications, there is a group of aesthetic and functional adverse effects associated with different muscle responses to botulinum toxin or with the misplacement of botulinum toxin. They are specific for botulinum toxin and are an effect of the direct pharmacological action of the toxin. They occur 
less frequently than injection reactions, and are primarily caused by temporary denervation of adjacent muscles outside of the intended treatment area. These complications are technique-dependent, because they are usually due to misplacement of the toxin or to different muscle reactivity. When adjacent muscles are inadvertently paralyzed, both aesthetic and functional problems may arise. There are some well-known, mild side effects of botulinum toxin treatment on the upper face. Most are self limiting with time. Common adverse effects can occur due to percutaneous injections on the forehead. The most common complication in the treatment of the glabellar complex is ptosis of the upper eyelid known as blepharoptosis. Its frequency is estimated at 1-5\% [1]. According to Vartanian and Dayan, blepharoptosis rates with forehead injections can range from $2 \%$ to $20 \%$ [6]. It is almost always unilateral and temporary, seen as a 2to $3-\mathrm{mm}$ lowering of the affected eyelid that is most marked at the end of the day with muscle fatigue [8]. Eyelid ptosis is a significant risk if injections are placed on or under the middle part between the eyebrows in the region of the midpupillary line. This is caused by diffusion of the toxin through the orbital septum fascia to the levator palpebrae superioris, an upper eyelid levator muscle. Injections of the orbicularis oculi, corrugator supercilii and procerus muscles have the highest likelihood of producing lid ptosis [6]. This is especially frequent in patients who have pre-existing lid ptosis or levator function weakness and in the elderly. Blepharoptosis is also the most common side effect of treating strabismus with botulinum toxin injections in children [11]. In the older patient, a combination of loose skin and attenuated orbital septum can further facilitate the dissection of injected botulinum toxin. The levator palpebrae muscle is particularly susceptible to low doses of botulinum toxin given its comparatively limited number of motor endplates [6]. The incidence of blepharoptosis is reduced by administering botulinum toxin injections at least $1 \mathrm{~cm}$ above the supraorbital ridge on the midpupillary line when treating the corrugator muscles. It can occur as early as $48 \mathrm{~h}$ or as late as 7-10 days following injection and can persist for 2-4 weeks. If ptosis occurs, it can be treated using ophthalmic solutions that have alpha-adrenergic effects, such as over-the-counter naphazoline $0.025 \%$ /pheniramine $0.3 \%$ or prescription apraclonidine $0.5 \%$. These mydriatic agents are alpha-adrenergic agonists and both cause contraction of Muller's muscle, thereby producing 1-2 $\mathrm{mm}$ elevation of the eyelid. The treatment is symptomatic and 1-2 drops three times a day must be continued until ptosis resolves. Apraclonidine is reserved for refractory cases and should be used with caution, because it can exacerbate or unmask underlying glaucoma [8]. Another periorbital complication related to botulinum toxin injections is ectropion. It is a technique-dependant complication that presents mainly in elderly patients. Periorbital injections that may be well tolerated in a younger patient may result in ectropion in the older patient. The development of postinjection ectropion requires prompt attention to prevent exposure keratitis and corneal damage. Topical lubricating drops, lid taping, and ocular moisture chambers may be helpful in the immediate period. An ophthalmologic consultation may be indicated [6]. Diplopia and transient strabismus are well-documented side effects of botulinum toxin injections in the lateral periorbital area. Diplopia that results from a crow's feet area injection is secondary to botulinum toxin diffusion leading to lateral rectus weakness. According to Kothari et al., diplopia occurs in $2.1 \%$ of patients [12]. It is mostly associated with inferior oblique paresis or rarely with lateral rectus paresis [12]. Potential risk factors for diplopia are inherent susceptibility, proximity of the needle tip to the trochlea, deep penetration of the needle into the orbital septum and increased diffusion of the drug following repeated injections [12]. Both complications need a consultation with an ophthalmologist to establish an accurate diagnosis and a temporary strabismus treatment plan. Eye patching or the application of a Fresnel membrane prism to eyeglasses can allay the diplopia until recovery [6]. Other periorbital complications are pseudoherniation of infraorbital fat pads, lagophthalmos and dry eye. The prominence of pseudoherniating infraorbital fat pads can occur following botulinum toxin treatments of the inferior eyelid in patients who have existing fat herniation. Compromise of orbicularis oculi tone in the patient who has lax septal support can result in the temporary herniation of infraorbital fat. Lagophthalmos is observed in patients who receive botulinum toxin injections for blepharospasm. Injecting larger quantities of botulinum toxin into the lateral canthal area may compromise orbicularis functions to the point of weakening eye closure and causing secondary dry eyes [6]. In rare cases, botulinum toxin injections may result in intraorbital or eye side effects. They include blurred vision, accommodation difficulties, retinal detachment, corneal irritation and corneal exposure. The visual symptoms can develop between 4 days and 2 weeks after the botulinum toxin injections and last between 6 weeks and 3 months. Usually, the patient's vision completely returns to baseline. The mechanism by which botulinum toxin injections cause visual symptoms is most likely due to the toxin's acetylcholine (Ach) blocking effects on parasympathetic nerve terminals in the visual system acquired by means of systemic spread. The parasympathetic nervous system, through the release of Ach, causes a contraction of the iris sphincter muscle for pupillary contraction $[13,14]$. It is possible that the effects of botulinum toxin remain local in the target muscle at lower doses, but systemic anticholinergic effects of the drug can be seen at higher doses or when in closer proximity to vascular structures at the injection sites $[13,15,16]$. Treatment of glabellar rhytides with botulinum toxin can result in lagophthal- 
mos and direct paralysis of the lacrimal gland, leading to dry eyes and superficial punctate keratitis [17]. Periorbital botulinum toxin injection can cause mydriasis of the pupil, resulting in acute angle closure attack. Cases have been reported in which the botulinum toxin was improperly injected into the vitreous cavity of a patient receiving treatment to the right medial rectus, resulting in increased intraocular pressure, decreased visual acuity and retinal detachment [18]. Apart from periorbital complications, other aesthetic side effects associated with botulinum toxin injection for forehead horizontal lines are the exaggeration of wrinkles, brow ptosis, "Mephisto sign", and periorbital edema. Paralysis of the frontalis is limited to the upper part of the frontalis and compensatory hyperactivation of the lower part of the frontalis muscle is possible in the lateral part of the forehead. Therefore, especially in people frequently using the frontalis muscle to raise their eyebrows or eyelids, exaggeration of previously unidentifiable wrinkles can take place on the border between the paralyzed frontalis muscle and nonparalyzed frontalis muscle. This complication is usually self-limiting and disappears spontaneously without any further treatment. For the compliance and satisfaction of patients, an additional botulinum toxin injection into hyperactivated muscles can be performed. The most significant complications of treatment of the frontalis are brow ptosis and undesired eyebrow shape. These complications can be corrected with a botulinum toxin injection into muscles that antagonize the affected muscles; however, complications caused by the involvement of adjacent muscles are temporary and will spontaneously resolve as the botulinum toxin effects diminish. They often result from overaggressive treatment with injections being administered too low on the forehead, or from poor patient selection. Treatment of the brow depressors can elevate the brow by 1-2 mm. Another unexpected change in eyebrow morphology is the "Mephisto sign". After injection, the distal ends of the eyebrows, which are normally positioned below the height of the eyebrow contour, become raised so that they are in a more superior position compared to the rest of the eyebrow, pointing upwards, forming the Mephisto sign. This condition occurs when the lateral fibers of the frontalis muscles are not sufficiently paralyzed compared to the medial fibers. The probability of the occurrence of the Mephisto sign may vary depending on the length, structure and morphology of the frontalis muscle and injection skills of the clinician. This effect may last at least 3 months. In the treatment or prevention of the Mephisto sign we can use an additional dose of botulinum toxin to paralyze lateral fibers of the frontalis muscle [19]. Another complication is an asymmetric smile caused by injection into the zygomaticus major. The injection should not be close to the inferior margin of the zygoma to avoid lip ptosis. Many of the muscles in the lower central face, especially those used in facial expressions, are also involved in the func- tions of the mouth and cheeks. The necessity for complete oral competence may result in botulinum toxin lip treatments being contraindicated in scuba divers, wind instrument players, and professional vocalists. Peng et al. reported a few rare complications, such as smile limitation, paradoxical bulging, sunken cheeks, and sagging after treatment of masseter muscle prominence or hypertrophy with botulinum toxin. Asymmetry in facial expressions after this procedure can be the result of the differences between the left and right masseters and the patient's unilateral preference in chewing and eating, and a subsequent failure to adjust the dosage accordingly. Different layers of the same muscles with opposite fiber direction may respond differently to the toxin. Muscular bulging during mastication is due to a superficial overcompensation of masseter muscle fibers in response to the neurotoxic weakening of the deep masseter [20]. Higher zygomas and less prominent cheek fat pads predispose to sunken lateral cheeks. Another uncommon complication of the cosmetic use of botulinum toxin in the lower face is difficulty in opening the mouth, resulting from paralysis of the lateral pterygoid muscles. Iatrogenic paralysis of the lateral pterygoid muscle is treated by mechanotherapy. Toxin diffusion to the parotid gland through the posterior part of the masseter muscle is due to xerostomia, requiring the use of sialagogues [20]. Dry mouth is also a side effect of treating Frey's syndrome with botulinum toxin [21].

The cosmetic use of Botox is safe even in cases of repeated injections. On the other hand, the long-lasting cosmetic use of botulinum toxin can trigger permanent changes in facial expression, and an expressionless, mask-like face. Lack of facial animation or permanent changes in facial expression may be undesirable in some cases. For individuals who have a greater need for facial animation, who must communicate with children, for actors, and broadcasters, such an outcome may have negative professional consequences. In accordance with this, such patients should be undertreated and the physician's strategy for a balanced botulinum toxin treatment should be shared with the patient.

\section{Serious adverse effects}

The potentially serious events are sequelae due to the systemic spread of toxin leading to botulism-like features or systemic anaphylactic reactions. The cosmetic use of botulinum toxin rarely causes severe side effects. The frequency of serious side effects is 33 times higher for therapeutic than for cosmetic cases. The median botulinum toxin dose is 4 times higher in reports for therapeutic cases than cosmetic cases [22]. The US Food and Drug Administration (FDA) describes adverse effects as serious if they meet the strict criteria. They are classified as a life-threatening experience requiring intervention to prevent permanent damage and associated with patient 
hospitalization or prolongation of existing hospitalization, and persistent or significant disability [22]. In 2005, the FDA reported 36 adverse effects related to cosmetic use, which met the FDA's definition of serious adverse events, with the majority concerning dysphagia [6]. Serious adverse effects after cosmetic use of botulinum toxin include dysphagia, muscle weakness, and allergic reactions [22, 23]. Dysphagia and muscle weakness are common symptoms of botulism. Cosmetic treatment of cervical wrinkles and lines usually requires larger doses of botulinum toxin and increases the risk of serious side effects. Platysmal injections in large doses (50 units and higher) to correct prominent vertical bands and horizontal neck lines may cause weakness of the neck flexors and temporary dysphagia, and, in rare cases, hoarseness. Older patients and larger doses increase the possibility of direct botulinum toxin injections or localized diffusion into the deeper cervical structures. Botulinum toxin can spread to a distance of 30-45 $\mathrm{mm}$ from the injected muscles to the adjacent muscles, even if these muscles are separated by fasciae. Neck muscles are prone to diffusion of botulinum toxin, and paralysis of the wrong muscles leads to dysphonia, dysphagia or dystonia. Mild and transient cervical discomfort usually occurs 2 to 5 days after injection. Dysphagia occurs on average 9.7 days after the injection and lasts on average 3.5 weeks. Clinically significant dysphagia resolves spontaneously within 2 weeks. If severe dysphagia is noted, a temporary change to a soft diet may be instituted and emotional support provided until full recovery occurs. Patients with severe dysphagia require nasal feeding or intravenous nutrition to maintain the water-electrolyte balance. One of the most common serious adverse effects of botulinum toxin is muscle weakness. This may involve injected muscles as well as adjacent muscles, or it may manifest as generalized muscle weakness. This weakness recurred a year after the first injection [24, 25]. This wide spectrum of symptoms associated with muscle weakness or paralysis, dysarthria, dysphonia, dysphagia and respiratory arrest is known as botulism. The first symptoms of botulism may be severe and long-lasting headache and fatigue. Some researchers report that up to $1 \%$ of patients who receive botulinum toxin injections may experience severe, debilitating headaches [6]. According to Bai et al., botulism can be classified into mild botulism with developing dizziness, headache, fatigue, blepharoptosis, and/or blurred vision, not affecting normal life; moderate botulism that additionally manifests dysphagia and/or bucking, and nasal feeding is adopted accordingly; and severe botulism, when apart from all of the aforementioned symptoms, respiratory failure appears and mechanical ventilation is applied [26]. Botulism is dose-dependent and results from the diffusion of the botulinum toxin into adjacent muscles from the injected muscles. It is interesting that botulism can occur even a few years after continued injections. Usually, toxic effects of botulinum toxin can appear at the $10^{\text {th }}$ or $11^{\text {th }}$ injection, after prior uncomplicated injections. The clinical symptoms of botulism after cosmetic use occur 0 to 36 days after injection, mainly between the $2^{\text {nd }}$ and $6^{\text {th }}$ day following the operation [26]. Differential diagnosis is conducted to discriminate botulism from myasthenia gravis, Guillain-Barré syndrome, cerebrovascular diseases, cervical vertebra diseases, and polymyositis. Treatment is based on the administration of neurotrophic drugs, systemic support and symptomatic treatment, and the injection of botulinum antitoxin serum. In cases of moderate to severe botulism, vital signs should be monitored closely. Severe-case patients should accept tracheal intubation and mechanical ventilation. Patients with a negative skin test should receive $10000 \mathrm{IU}$ of antitoxin serum via intramuscular injection once every $12 \mathrm{~h}$; patients with a positive or weak positive skin test should receive 10000 IU via desensitization injection once a day [26]. Early application of botulinum antitoxin serum and certain symptomatic support treatments, such as active infection prevention, is important for botulism treatment. The earlier the treatments are performed, the better the effects are [26]. The mechanism responsible for the generalized diffusion of botulinum toxin is not known. Hypotheses proposed concern either systemic spread or retrograde axonal spread of toxin. It is not clear whether the generalized diffusion is due to the spread of botulinum toxin or of its inactive metabolites [27]. The toxin may be able to traverse through an intact vessel wall. In addition, accidental intravenous delivery of the toxin may occur when it is injected into muscle [28]. Diffusion of botulinum toxin to contralateral muscles has also been reported. Moreover, experimental studies have shown that botulinum toxin receptors exist in the central nervous system and a small amount of botulinum toxin crosses the blood-brain barrier. This raises the possibility that botulinum toxin is transported retrogradely, similarly to tetanus toxin, and may cause centrally mediated side effects. Another theory postulated relates to absorption of a small amount of botulinum toxin into the bloodstream and its systemic action [4]. In addition to the mechanism of generalized diffusion, another source of severe side effects is anaphylactic shock. It is attributed to the chemical structure of the drug, resulting in hypersensitivity reactions. Using standard emergency protocols and medications such as epinephrine and methylprednisolone is advised when indicated, rather than diphenhydramine, because of its anticholinergic effects. There is no difference in the frequency of anaphylactic shock between the cosmetic and therapeutic use of botulinum toxin [29]. Repeated injections cause immune complexes to form in the botulinum toxin, which induces blocking of antibodies. Other immune-mediated reactions are the source of both hypersensitivity and immunoresistance to botulinum toxin. Some reports indicate that antibotulinum toxin A antibodies can occur in $5 \%$ to 
Table 1. The most common side effects related to cosmetic use of botulinum toxin

\begin{tabular}{|c|c|c|c|}
\hline $\begin{array}{l}\text { Mechanisms of side } \\
\text { effects }\end{array}$ & Type of side effects & Prevention & Treatment \\
\hline \multicolumn{4}{|l|}{ Injection site side effects: } \\
\hline Injuring a blood vessel & $\begin{array}{l}\text { Erythema, ecchymosis } \\
\text { Bruising, hematoma }\end{array}$ & Thorough medical history & Tamponade, pressure, ice packs \\
\hline $\begin{array}{l}\text { The needle puncturing } \\
\text { the skin }\end{array}$ & $\begin{array}{l}\text { Tenderness and pain at the site of } \\
\text { injection, injection trauma }\end{array}$ & $\begin{array}{l}\text { Topical anesthetic creams, } \\
\text { correct needle size }\end{array}$ & Topical anesthetic creams \\
\hline $\begin{array}{l}\text { Decreased sweat } \\
\text { gland activity }\end{array}$ & Dry skin & No recommendations & Emollients \\
\hline $\begin{array}{l}\text { Contamination of the } \\
\text { injection site }\end{array}$ & Local infection, abscess & Aseptic technique & Course of antibiotics \\
\hline Nerve injures & Paresthesia or dysesthesia & No recommendations & No specific treatment \\
\hline \multicolumn{4}{|c|}{ Technique-dependent side effects: } \\
\hline $\begin{array}{l}\text { Aesthetic in } \\
\text { periorbital area }\end{array}$ & $\begin{array}{l}\text { "Mephisto sign", exaggeration of wrinkles, } \\
\text { brow ptosis, periorbital edema }\end{array}$ & Correct dose and technique & Paralysis of antagonist muscles \\
\hline $\begin{array}{l}\text { Functional in } \\
\text { periorbital area }\end{array}$ & $\begin{array}{l}\text { Blepharoptosis, ptosis } \\
\text { Sctropion, dry eyes, corneal irritation, } \\
\text { corneal exposure } \\
\text { Blurred vision, accommodation difficulties, } \\
\text { retinal detachment, diplopia, strabismus }\end{array}$ & Correct technique & $\begin{array}{c}\alpha \text {-Adrenergic drops } \\
\text { Topical lubricating drops } \\
\text { Ophthalmologic consultation }\end{array}$ \\
\hline $\begin{array}{l}\text { Aesthetic in perioral } \\
\text { area }\end{array}$ & $\begin{array}{l}\text { Asymmetric smile, lip ptosis, changes in } \\
\text { facial expression }\end{array}$ & Correct technique & No specific treatment \\
\hline \multirow{2}{*}{$\begin{array}{l}\text { Functional in perioral } \\
\text { area }\end{array}$} & Trismus & Correct technique & Mechanotherapy \\
\hline & Xerostomia & Correct technique & Sialagogues \\
\hline \multicolumn{4}{|l|}{ Allergic reactions: } \\
\hline Localized & Redness, edema, urticaria, & Thorough medical history & $\begin{array}{l}\text { Antihistamine drugs, local } \\
\text { steroids }\end{array}$ \\
\hline Generalized & $\begin{array}{l}\text { Generalized urticaria, diffuse edema, } \\
\text { anaphylactic shock }\end{array}$ & Thorough medical history & $\begin{array}{l}\text { Steroids, adrenalin injection, } \\
\text { monitoring of patients }\end{array}$ \\
\hline Other mechanism & Headache & No specific prevention & Analgesics \\
\hline $\begin{array}{l}\text { Diffuse spread of toxin } \\
\text { (botulism) }\end{array}$ & $\begin{array}{l}\text { Severe dysphagia } \\
\text { Generalized muscle weakness }\end{array}$ & $\begin{array}{l}\text { Minimal dose, correct } \\
\text { technique } \\
\text { Minimal dose, correct } \\
\text { technique }\end{array}$ & $\begin{array}{l}\text { Neurotrophic drugs, systemic } \\
\text { support and symptomatic } \\
\text { treatment botulinum antitoxin } \\
\text { serum }\end{array}$ \\
\hline
\end{tabular}

$10 \%$ of patients who receive repeated, long-term, highdose botulinum toxin treatments. Other types of BoNT-A have different antigenicity. It was reported that incobotulinumtoxinA (Xeomin) is characterized by reduced antigenicity [30]. Antibody development against various components of botulinum toxin complex is evidence of systemic botulinum toxin exposure. The factors that contribute to antibody formation include longer duration of treatment, a shorter time interval between injections, larger overall doses, and decreased purity of botulinum toxin preparation [6]. Formation of antibodies is estimated at less than 1\% [7]. Immunoresistance is another entity that has been clinically described in selected patients, especially those injected with large doses in multiple treatment sessions [4]. Clinically significant botulinum toxin resistance is less common in patients who receive the lower doses of botulinum toxin that are typically administered in cosmetic treatments. On the other hand, immunoresistance in the cosmetic use of botulinum toxin results in a higher risk of dose-dependent serious side effects. Immunoresistance develops within the first years of therapy. If immunoresistance to botulinum toxin is not noted within the first 4 years, then it is unlikely to develop. Those who develop immunoresistance can be treated with breakthrough doses of botulinum toxin, or with nontype A botulinum toxin, such as botulinum toxin B [6]. The lack of sensitivity to botulinum toxin can be attributed to a number of reasons, from inappropriate diagnosis to inappropriate target muscle selection or injection, or from the use of an amount of botulinum toxin insufficient to elicit the desired pharmacological outcome to the presence of antitoxin-neutralizing antibodies. Some research has excluded the possible role of mutations affecting either binding or substrate 
cleavage of botulinum toxin in the mechanisms of primary resistance to botulinum toxin [31]. Repeated injections may eventually enhance the pathological innervation, leading to tolerance and even exacerbation of local symptoms. Moreover, they cause muscle fibrosis after several years, though such an effect has not been shown in shorter follow-up so far. Furthermore, medications that inhibit neuromuscular signaling, such as aminoglycosides, quinidine, anticholinergics and muscle relaxants, may potentiate botulinum toxin effects [7]. Serious adverse events related to the cosmetic use of botulinum toxin include thyroid eye disease in a patient with Graves hyperthyroidism, sarcoidal granuloma, pseudoaneurysm of the frontal branch of the superior temporal artery, and severe respiratory failure. It seems that cosmetic use of botulinum toxin is not time-dependant. There is no evidence of cumulative adverse events after cosmetic use of botulinum toxin. Moreover, the event rate decreases in later treatment cycles. Furthermore, there are no severe adverse effects in patients who regularly repeat botulinum toxin injections for cosmetic indications (Table 1).

\section{Prevention of side effects}

There are a few potentially important factors for the prevention of side effects after botulinum toxin use. First of all, physicians should establish indications for these procedures properly. They should be familiar with the anatomy of the muscles involved and with any alterations to the anatomy due to prior surgical procedures. Thorough knowledge of the anatomy of the muscles being treated is imperative to avoid serious adverse events, especially those resulting from generalized diffusion. Altered anatomy as a result of previous plastic facial surgery predisposes to more severe botulinum toxin-related side effects. Proper storage of the product, selection of the correct dose, and proper reconstitution and administration techniques are important. Selected adverse effects in cosmetic use of botulinum toxin may be limited by modifications of the technique. Intradermal injection of botulinum toxin has a similar anti-wrinkle efficacy compared to intramuscular injection, but is characterized by a lower risk of eyebrow ptosis, and by eyebrow heaviness. This modification may be effective and safe for reducing wrinkles on the forehead [15]. The appropriate technique decreases the risk of periorbital complications. Direct digital pressure can be used to "isolate" the injection site from the eye. In addition, when injecting the glabellar area, the corrugator muscle can be grasped between two fingers to decrease regional extravasation. A useful guideline for reducing blepharoptosis is to place corrugator injections at least $1 \mathrm{~cm}$ above the level of the supraorbital ridge [5]. Botulinum toxin injection outside the "orbital zone" is recommended to minimize complications. Keeping all injections outside the boundary defined by the infraorbital rim and a point $1 \mathrm{~cm}$ lateral to the lateral canthus can significantly reduce the risk of ectropion. In the periorbital area the recommended distances should be kept. The needle should always point away from the orbit to avoid penetrating the septum, which serves as a diffusion barrier [32]. Most of these complications are dose-dependent, and a lower dose of botulinum toxin decreases their frequency. Moreover, the median lethal dose of botulinum toxin used at present to estimate safety in humans is a measure only of acute drug lethality and does not reflect acute or chronic drug toxicity, for example, to the brain or other organs, which can occur at lower doses or with chronic drug administration. It is difficult to predict the dose-dependent possible side effect after botulinum toxin use. Pharmacokinetics of botulinum toxin may be different in different organs [27]. Underdosage is always recommendable. As the individual reaction is not always predictable, a cautious approach with rather low dosages is advisable when treating a patient for the first time. In perioral treatments, only the smallest doses are needed. A slightly higher dosage may lead to difficulties in drinking, whistling and talking $[33,34]$. Although the maximum dose per treatment session for glabellar lines is $20 \mathrm{U}$ for all sessions combined, the upper dosing limit for the use of botulinum toxin has not been specified. Multiple injections of lower doses of botulinum toxin appear to play a role in achieving a satisfactory result and in preventing systemic side effects. Multiple injections of lower doses reduce this risk. Many studies recommend the dosing of botulinum toxin based on fat-free mass or on body weight. Another important factor can be the interval between injections. Botulinum toxin binds to specific receptors irreversibly at the nerve terminal. With repeated injections, the receptor-binding capacity may be exceeded, which can lead to systemic spread. To prevent the accumulation of botulinum toxin on failure of the treatment, a certain interval between successive injections is to be respected. Dilution of botulinum toxin and the number of muscles injected are considered to be important factors for avoiding serious adverse effects. In general, botulinum toxin is contraindicated in patients with a known hypersensitivity to the components of the formulation or with disorders of the neuromuscular junction. Patients taking aminoglycoside antibiotics or anticoagulants should not be treated with botulinum toxins. Patients with diseases of the neuromuscular junction, such as myasthenia gravis, multiple sclerosis and Lambert-Eaton syndrome, are particularly susceptible to the adverse events of botulinum toxin. Patients with anterior horn disorders are also reported to have hypersensitivity to botulinum toxin. In any state where neuromuscular transmission is compromised, botulinum toxin injections may potentially worsen symptoms of the existing disease state [6]. Botulinum toxin treatments are not administered during pregnancy or while nursing. There are no reports of teratogenic effects in humans from in utero botulinum toxin exposure. How- 
ever, there are accounts of women who, while unaware of being pregnant, received a botulinum toxin injection without experiencing pregnancy-related problems. Nevertheless, the FDA classifies botulinum toxin as a category $C$ drug, indicating that its safety profile during pregnancy has not been adequately evaluated [6]. Until additional evidence supporting the safe use of botulinum toxin during pregnancy and nursing is presented, pregnant women and nursing mothers should not be treated [6]. Aesthetic botulinum toxin application in patients older than 75 years is controversial, because of the higher risk of undiagnosed neurologic and medical disorders, and the greater likelihood of drug therapy interactions. Taking a thorough medical patient's history helps to avoid most of the potential side effects that follow botulinum toxin administration. There are a few possible botulinum toxin-drug interactions which can modify the final effect of injections and trigger unexpected complications. Drugs that may alter the effects of botulinum toxin include aminoglycosides, cyclosporine, D-penicillamine, muscle relaxants, especially curare-type nondepolarizing blockers and succinylcholine, aminoquinolones, quinidine, magnesium sulfate, and lincosamide. Large doses of aminoglycosides can prevent the release of acetylcholine into the neuromuscular junction and induce a botulism-like clinical state. Cyclosporine can induce muscle weakness secondary to neuromuscular blockade, and can lead to respiratory failure. Discontinuing therapy with these drugs or a short cessation is recommended to avoid potential botulinum toxin-related side effects [34-36].

\section{Acknowledgments}

The study was conducted in the Department of Plastic, Reconstructive and Aesthetic Surgery, Nicolaus Copernicus University, Torun.

\section{Conflict of interest}

The authors declare no conflict of interest.

\section{References}

1. Yiannakopoulou E. Serious and long-term adverse events associated with the therapeutic and cosmetic use of botulinum toxin. Pharmacology 2015; 95: 65-9.

2. Field M, Splevins A, Picaut $P$, et al. AbobotulinumtoxinA (Dysport $\left.{ }^{\circledR}\right)$, OnabotulinumtoxinA (Botox ${ }^{\circledR}$ ), and IncobotulinumtoxinA (Xeomin ${ }^{\circledR}$ ) neurotoxin content and potential implications for duration of response in patients. Toxins 2018; 10: 535.

3. Omprakash HM, Rajendran SC. Botulinum toxin deaths: what is the fact? J Cutan Aesthet Surg 2008; 1: 95-7.

4. Roy D, Sadick NS. Complications of botulinum toxin. Compl Cutan Surg 2018: 207-12.

5. Shetty MK. Guidelines on the use of botulinum toxin type A. Indian J Dermatol Venereol Leprol 2008; 74 Suppl: S13-22.
6. Vartanian AJ, Dayan SH. Complications of botulinum toxin A use in facial rejuvenation. Facial Plast Surg Clin North Am 2005; 13: 1-10.

7. Ward NL, Kavlick KD, Diaconu D, et al. Botulinum neurotoxin A decreases infiltrating cutaneous lymphocytes and improves acanthosis in the KC-Tie2 mouse model. J Invest Dermatol 2012; 137: 1927-30.

8. Small R. Botulinum toxin injection for facial wrinkles. Am Fam Physician 2014; 90: 168-75.

9. Park KY, Hyun MY, Jeong SY, et al. Botulinum toxin for the treatment of refractory erythema and flushing of rosacea. Dermatology 2015; 230: 299-301.

10. Bloom BS, Payongayong L, Mourin A, et al. Impact of intradermal abobotulinumtoxin A on facial erythema of rosacea. Dermatol Surg 2015; 41 Suppl 1: S9-16.

11. Rowe F, Noonan C. Complications of botulinum toxin a and their adverse effects. Strabismus 2009; 17: 139-42.

12. Kothari M, Shukri N, Quayyum A. Transient superior oblique paresis after injection of botulinum toxin A for facial rejuvenation. Indian J Ophthalmol 2012; 60: 77-8.

13. Dubow J, Kim A, Leikin J, et al. Visual system side effects caused by parasympathetic dysfunction after botulinum toxin type B injections. Mov Disord 2005; 20: 877-80.

14. Kalra HK, Magoon EH. Side effects of the use of botulinum toxin for treatment of benign essential blepharospasm and hemifacial spasm. Ophthalmic Surg 1990; 21: 335-8.

15. Kang SM, Feneran A, Kim JK, et al. Exaggeration of wrinkles after botulinum toxin injection for forehead horizontal lines. Ann Dermatol 2011; 23: 217-21.

16. Jun JY, Park JH, Youn CS, et al. Intradermal injection of botulinum toxin: a safer treatment modality for forehead wrinkles. Ann Dermatol 2018; 30: 458-61.

17. Northington ME, Huang CC. Dry eyes and superficial punctate keratitis: a complication of treatment of glabelar dynamic rhytides with botulinum exotoxin A. Dermatol Surg 2004; 30: 1515-7.

18. Lee DH, Han J, Han SH, et al. Vitreous hemorrhage and Rhegmatogenous retinal detachment that developed after botulinum toxin injection to the extraocular muscle: case report. BMC Ophthalmol 2017; 17: 249.

19. Cho ES, Hwang JY, Kim ST. A proposal to prevent the "Mephisto sign" side effect to botulinum toxin type A injection in chronic migraine. Yonsei Med J 2013; 54: 1542-4.

20.Peng HP, Peng JH. Complications of botulinum toxin injection for masseter hypertrophy: incidence rate from 2036 treatments and summary of causes and preventions. J Cosmet Dermatol 2018; 17: 33-8.

21. Xie S, Wang K, Xu T, et al. Efficacy and safety of botulinum toxin type A for treatment of Frey's syndrome: evidence from 22 published articles. Cancer Med 2015; 4: 1639-50.

22. Coté TR, Mohan AK, Polder JA, et al. Botulinum toxin type A injections: adverse events reported to the US Food and Drug Administration in therapeutic and cosmetic cases. J Am Acad Dermatol 2005; 53: 407-15.

23. Paget SP, Swinney CM, Burton KLO, et al. Systemic adverse events after botulin neurotoxin A injections in children with cerebral palsy. Dev Med Child Neurol 2018; 60: 1172-7.

24. Varghese-Kroll E, Elovic EP. Contralateral weakness and fatigue after high-dose botulinum toxin injection for management of poststroke spasticity. Am J Phys Med Rehabil 2009; 88: 495-9.

25. Thomas AM, Simpson DM. Contralateral weakness following botulinum toxin for poststroke spasticity. Muscle Nerve 2012; 46: 443-8. 
26. Bai L, Peng X, Liu Y, et al. Clinical analysis of 86 botulism cases caused by cosmetic injection of botulinum toxin (BoNT). Medicine (Baltimore) 2018; 97: e10659.

27. De Leat K, Wyndaele JJ. Adverse events after botulinum A toxin injection for neurogenic voiding disorders. Spinal Cord 2005; 43: 397-9.

28. Hristova AH, Joseph LN, Sathe SA, et al. Severe nervous system complications after botulinum type A therapy: three case reports with reviews of FDA-reported nervous system adverse effects. PMR 2012; 4: 613-23.

29. Li M, Goldberger BA, Hopkins C. Fatal case of BOTOX-related anaphylaxis? J Forensic Sci 2005; 50: 169-72.

30. Dressler D. Five-year experience with incobotulinumtoxin A $\left(\right.$ Xeomin $\left.\left({ }^{\circledR}\right)\right)$ : the first botulinum toxin drug free of complexing proteins. Eur J Neurol 2012; 19: 385-9.

31. Pirazzini M, Carle S, Barth $\mathrm{H}$, et al. Primary resistance of human patients to botulinum neurotoxins A and B. Ann Clin Transl Neurol 2018; 5: 971-5.

32. Sommer B. How to avoid complications when treating hyperdynamic folds and wrinkles. Clin Dermatol 2003; 21: 521-3.

33. Suputtitada A, Phanthumchinda K, Locharernkul C, et al. Hemifacial spasm: results of treatment with low dose botulinum toxin injection. J Med Assoc Thai 2004; 87: 1205-11.

34. van Hulst K, Kouwenberg CV, Jongerius PH, et al. Negative effects of submandibular botulinum neurotoxin $A$ injections on oral motor function in children with drooling due to central nervous system disorders. Dev Med Child Neurol 2017; 59: 531-7.

35. Karbassi E, Nakhaee N, Zamanian M. The efficacy and complications of a new technique of Abobotulinum-toxin A (Dysport) injection in patients with glabellar lines. J Cosmet Dermatol 2018; 23. doi: 10.1111/jocd.12529.

36. Bakheit AM, Ward CD, McLellan DL. Generalised botulismlike syndrome after intramuscular injections of botulinum toxin type A: a report of two cases. J Neurol Neurosurg Psychiatry 1997; 62: 198. 